\title{
A HARNACK INEQUALITY FOR HOMOGENEOUS GRAPHS AND SUBGRAPHS
}

\author{
F. R. K. CHUNG AND S.-T. YAU
}

\begin{abstract}
We prove a Harnack inequality for eigenfunctions of certain homogeneous graphs and subgraphs which we call strongly convex. This inequality can be used to derive a lower bound for the (nontrivial) Neumann eigenvalues by $1 /\left(8 k D^{2}\right)$ where $k$ is the maximum degree and $D$ denotes the diameter of the graph.
\end{abstract}

\section{INTRODUCTION}

Let $\Gamma=(V, E)$ denote a graph with vertex set $V=V(\Gamma)$ and edge set $E=E(\Gamma)$. Suppose a group $\mathcal{H}$ acts on $V$ such that:

(i) for all $g \in \mathcal{H},\{g u, g v\} \in E$ if and only if $\{u, v\} \in E$,

(ii) for any two vertices $u$ and $v$, there is a $g \in \mathcal{H}$ such that $g u=v$.

Then we say $\Gamma$ is a homogeneous graph with the associated group $\mathcal{H}$. In other words, $\Gamma$ is vertex-transitive under the action of $\mathcal{H}$ and We can identify $V$ with the coset space $\mathcal{H} / \mathcal{I}$ where $\mathcal{I}=\{g \in \mathcal{H}: g v=v\}$, for a fixed vertex $v$, denotes the isotropy group. We note that the Cayley graph is a special case of homogeneous graphs with $\mathcal{I}$ trivial. The edge set of a homogeneous graph $\Gamma$ can be described by an (edge) generating set $K \subset \mathcal{H}$ so that each edge of $\Gamma$ is of the form $\{v, g v\}$ for some $v \in V$, and $g \in K$. In this paper we require the generating set $K$ to be symmetric, i.e., $g \in K$ if and only if $g^{-1} \in K$.

We say that a homogeneous graph is invariant if for every element $a \in K$, we have

$$
a K a^{-1}=K
$$

In other words, $K$ is invariant as a set under conjugation by elements of $K$. For example, a homogeneous graph associated with an abelian group is invariant 
and we call the graph an abelian homogeneous graph. Suppose a homogeneous graph $\Gamma$ is associated with a nonabelian group $\mathcal{H}$. If the edge generating set $K$ is a subgroup of $\mathcal{H}$, then $\Gamma$ is still invariant.

In a graph $G$, the distance $d(u, v)$ between two vertices $u$ and $v$, is the minimum number of edges in any path joining $u$ and $v$ in $G$, and the diameter of $G$, denoted by $D(G)$ is the maximum distance $d(u, v)$ among all pairs of vertices.

The diameter is closely related to various graph invariants. Let $\lambda$ denote the dominant eigenvalue of the Laplacian of $G$. For a regular graph $G$, the Laplacian $L$ of $G$ is just $I-\frac{1}{k} A$ where $I$ is the identity matrix, $A$ is the adjacency matrix and $G$ is $k$-regular. The eigenvalues of $L$ are denoted by $0=\lambda_{0} \leq \lambda_{1} \leq \cdots \leq \lambda_{n-1}$. Many results for random walks on groups and Markov chains are based upon the following inequality for vertex-transitive graphs $[1,2,6]$.

$$
\lambda_{1} \geq \frac{c}{k D^{2}}
$$

where $c$ denotes some appropriate absolute constant, $k$ is the degree and $D$ denotes the diameter of the graph. For a general graph, however, (1) does not necessarily hold. (For example, the graph of two cliques $K_{n}$ joined by an edge has $\lambda \leq \frac{c}{n^{2}}$ and $D=3$ ). The main result of this paper is to generalize (1) for certain induced subgraphs of a homogeneous graph. An induced subgraph determined by a subset $S$ of the vertices has edge set consisting of all edges with both endpoints in $S$. For convenience, we will use $S$ to denote both the induced subgraph and its vertex set, if there is no confusion.

An induced subgraph of a homogeneous graph can be viewed as a graph with boundary while the "host" homogeneous graph has no boundary. In Section 2, we will define the Laplacian and Neumann eigenvalues for an induced subgraph of a homogeneous graph. This can be viewed as the discrete analogue corresponding to the continuous case of Neumann eigenvalues for Riemannian manifolds with boundary (which is different from the Dirichlet eignvalues for domains with boundary, cf. [7]). 
An induced subgraph $S$ of a graph $G$ is said to be strongly convex if, for all pairs of vertices $u$ and $v$ in $S$, all shortest paths joining $u$ and $v$ in $G$ are contained in $S$. We will show that for a finite strongly convex subgraph $S$ in an invariant abelian homogeneous graph $\Gamma$, an eigenfunction $f$ with eigenvalue $\lambda$ satisfies

$$
|f(x)-f(y)|^{2} \leq 8 k \lambda \sup _{z \in S}|f(z)|^{2}
$$

where the vertices $x$ and $y$ are adjacent and $k$ is the degree of $\Gamma$. Furthermore, the dominant eigenvalue $\lambda_{S}$ of the Neumann Laplacian of $S$ satisfies

$$
\lambda_{S} \geq \frac{1}{8 k D^{2}}
$$

where $D$ is the diameter of $S$.

This paper is organized as follows: In Section 2, we give definitions and examples for the Laplacian of a homogeneous graph, the Neumann eigenvalues of an induced subgraph of a homogeneous graph and strongly convex subgraphs. In Section 3, we derive a Harnack inequality for eigenfunctions of invariant homogeneous graphs. In Section 4, we prove a Harnack inequality for strongly convex subgraphs of invariant abelian homogeneous graphs. In Section 5 we establish an eigenvalue inequality using the Harnack inequality and then briefly discuss some applications of (1.2) in random walks and rapidly mixing Markov chains.

\section{The Laplacian, CONVEXity and Neumann eigenvalues}

Let $\Gamma$ denote a homogeneous graph with associated group $\mathcal{H}$ and an edge generating set $K$. Let $k=|K|$. The Laplacian $L$ of $\Gamma$ acts on the space of functions $f: V(\Gamma) \rightarrow \mathbf{R}$ as follows:

$$
L f(x)=\frac{1}{k} \sum_{g \in K}(f(x)-f(g x))
$$

and, therefore

$$
\langle f, L f\rangle=\frac{1}{k} \sum_{\substack{x, y \\ x \sim y}}(f(x)-f(y))^{2}
$$

Equivalently, we have

$$
L=I-\frac{1}{k} A
$$


where $A$ is the adjacency matrix of $\Gamma$. Various properties of Laplacians and eigenvalues for general graphs can be found in [3]

For an induced subgraph $S$ of a homogeneous graph $\Gamma$, we define the boundary $\delta S$ of $S$ by

$$
\delta(S)=\{v \in V \backslash S: v \sim u \in S\} .
$$

An induced subgraph $S$ of a homogeneous graph $\Gamma$ is said to be strongly convex if the edge generating set $K$ satisfies the following condition:

(A): For all $a, b \in K, x \notin \delta S$, if $a x \in S, b x \in S$, then we have $b^{-1} a \in K$.

We note that $(\mathrm{A})$ is implied by the following condition:

(B): For all pairs of vertices $u$ and $v$ in $S$, all shortest paths joining $u$ and $v$ are contained in $S$.

Clearly, the intersection of two strongly convex subgraphs is strongly convex. Example 1: Suppose we consider a homogeneous graph $\Gamma_{t}$ with vertex set $\left.\left\{\left(a_{1}, a_{2}, \cdots, a_{t}\right)\right\}: a_{i} \in \mathbf{Z}, \sum_{i} a_{i}=n\right\}$. A vertex $\left(a_{1}, \cdots, a_{i}, \cdots, a_{j}, \cdots, a_{t}\right)$ is adjacent to $\left(a_{1}, \cdots, a_{i}+1, \cdots, a_{j}-1, \cdots, a_{t}\right)$ for $1 \leq i, j \leq t$. In particular, for a fixed $i$, we define $\left.H_{i}=\left\{\left(a_{1}, a_{2}, \cdots, a_{t}\right)\right\}: a_{i} \geq 0\right\}$ to be a halfplane. It is easy to see that $H_{i}$ is strongly convex and $\cap H_{i}=\left\{\left(a_{1}, a_{2}, \cdots, a_{t}\right)\right\} \in V\left(\Gamma_{t}\right)$ : $\left.a_{i} \geq 0\right\}$ is strongly convex.

Let $S$ denote a finite induced subgraph of a homogeneous graph $\Gamma$. We define the extension $\hat{S}$ of $S$ to be the graph formed by all edges $\{x, y\}$ with at least one endpoint in $S$. We now define the Neumann eigenvalue of the subgraph $S$ as follows:

$$
\lambda_{S}=\inf _{f} \frac{\sum_{\{x, y\} \in \hat{S}}(f(x)-f(y))^{2}}{k \sum_{x \in S} f^{2}(x)}
$$

where $k$ denotes the degree in $\Gamma$ and $f$ ranges over all functions $f: \delta S \cup S \rightarrow \mathbf{R}$ satisfying

$$
\sum_{x \in S} f(x)=0
$$


Lemma 1. Suppose $f$ is the eigenfunction achieving $\lambda_{S}$ in (2.3). For $x \in S$, we have

$$
L f(x)=\frac{1}{k} \sum_{g \in K}[f(x)-f(g x)]=\lambda_{S} f(x)
$$

and for $x \in \delta S$, we have

$$
\sum_{\substack{g \in K \\ g x \in S}}[f(x)-f(g x)]=0
$$

The proof is by using a variational principle and will be omitted. We remark that the second equation in Lemma 1 corresponds to the vanishing of the normal derivative at the boundary which is usually called the Neumann condition.

We also remark that the Neumann eigenvalues are intimately related to problems arising in random walks. In an induced subgraph $S$ of a homogeneous graph with degree $k$, we define the following random walk: The probability of moving from a vertex $v$ to a neighbor $u$ of $v$ is $1 / k$ if $u$ is in $S$. If $u$ is not in $S$, we then move from $v$ to each neighbor of $u$ in $S$ with the (additional) probability $1 / k d_{u}$ where $d_{u}$ denotes the number of neighbors of $u$ in $S$. The transition matrix $P$ for this walk, whose columns and rows are indexed by $S$, is defined as follows:

$$
P f(v)=\sum_{\substack{u \in S \\ u \sim v}} \frac{1}{k} f(u)+\sum_{\substack{u \in S \\ u \sim \mathcal{\sim} \sim \widetilde{S} \\ z \notin \mathcal{S}}} \frac{1}{k d_{z}} f(u)
$$

It can be easily checked that the stationary distribution is the uniform distribution. Furthermore, the dominant eigenvalues $\rho$ of $P$ are related to the Neumann eigenvalues $\lambda_{S}$ by

$$
1-\rho=\lambda_{S}
$$


This can be proved by using the Neumann condition in Lemma 1 as follows:

$$
\begin{aligned}
1-\rho & =\inf _{f} \frac{\sum_{\substack{x \sim y \\
x, y \in S}}(f(x)-f(y))^{2}+\sum_{\substack{x \sim z \sim y \\
x, y \in S, z \notin S}}(f(x)-f(y))^{2} / d_{z}}{k \sum_{x \in S} f^{2}(x)} \\
& =\inf _{f} \frac{\sum_{\substack{x \sim y \\
x, y \in S}}(f(x)-f(y))^{2}+\sum_{z \notin S S} \sum_{\substack{x \sim z \\
x \in S}}\left[d_{z} f^{2}(x)-\left(\sum_{\substack{y \sim z \\
y \in S}} f(y)\right)^{2}\right] / d_{z}}{k \sum_{\substack{x \in S \\
x^{2}}} f^{2}(x)} \\
& =\inf _{f} \frac{\sum_{\substack{x \sim y \\
x, y \in S}}(f(x)-f(y))^{2}+\sum_{z \notin S} \sum_{\substack{x \sim z \\
x \in S}}\left(f^{2}(x)-f^{2}(z)\right)}{k \sum_{x \in S} f^{2}(x)} \\
& =\inf _{f} \frac{\sum_{\substack{x \sim y \\
x, y \in S}}(f(x)-f(y))^{2}+\sum_{z \notin S} \sum_{\substack{x \sim z \\
x \in S}}(f(x)-f(z))^{2}}{k \sum_{x \in S} f^{2}(x)} \\
& =\inf _{f} \frac{\sum_{\{x, y\} \in \hat{S}}(f(x)-f(y))^{2}}{k \sum_{x \in S} f^{2}(x)} \\
= & \lambda_{S}{ }^{2}
\end{aligned}
$$

where $f$ ranges over all functions $f: \delta S \cup S \rightarrow \mathbf{R}$ satisfying

$$
\sum_{x \in S} f(x)=0
$$

The inequality (2.5) is quite useful in bounding the rate of convergence of random walks and the rapidly mixing of Markov Chains. For more discussions on random walk problems and their relations with the Neumann eigenvalue $\lambda_{S}$, the reader is referred to [3].

\section{A HARNACK INEQUALITY FOR HOMOGENEOUS GRAPHS}

We will first prove the following Harnack inequality for homogeneous graphs which are invariant. 
Theorem 0. In an invariant homogeneous graph $\Gamma$ with edge generating set $K$ consisting of $k$ generators, suppose a function $f: V(\Gamma) \rightarrow \mathbf{R}$ satisfies

$$
L f(x)=\frac{1}{k} \sum_{a \in K}[f(x)-f(a x)]=\lambda f(x) .
$$

Then the following inequality holds for all $x \in V(\Gamma)$ :

$$
\frac{1}{k} \sum_{a \in K}[f(x)-f(a x)]^{2}+\alpha \lambda f^{2}(x) \leq \frac{\lambda \alpha^{2}}{\alpha-2} \sup _{y} f^{2}(y)
$$

for any $\alpha>2$.

Proof. We define

$$
\rho(x)=\frac{1}{k} \sum_{g \in K}[f(x)-f(g x)]^{2}
$$

and we consider

$$
\begin{aligned}
L \rho(x)= & \frac{1}{k^{2}} \sum_{b \in K} \sum_{a \in K}\left\{[f(x)-f(a x)]^{2}-[f(b x)-f(a b x)]^{2}\right\} \\
= & -\frac{1}{k^{2}} \sum_{b \in K} \sum_{a \in K}[f(x)-f(a x)-f(b x)+f(a b x)]^{2} \\
& +\frac{2}{k^{2}} \sum_{b \in K} \sum_{a \in K}[f(x)-f(a x)-f(b x)+f(a b x)][f(x)-f(a x)]
\end{aligned}
$$

Let $X$ denote the second term above and we have

$$
\begin{aligned}
X= & \frac{2}{k^{2}} \sum_{b \in K} \sum_{a \in K}[f(x)-f(a x)-f(b x)+f(a b x)][f(x)-f(a x)] \\
= & \frac{2}{k^{2}} \sum_{a \in K}\left\{\sum_{b \in K}[f(x)-f(a x)-f(b x)+f(b a x)]\right\}[f(x)-f(a x)] \\
& +\frac{2}{k^{2}} \sum_{a \in K}\left[\sum_{b \in K}(f(a b x)-f(b a x))\right][f(x)-f(a x)] \\
= & \frac{2 \lambda}{k} \sum_{a \in K}[f(x)-f(a x)]^{2}+\frac{2}{k^{2}} \sum_{a \in K}\left[\sum_{b \in K}(f(a b x)-f(b a x))\right][f(x)-f(a x)]
\end{aligned}
$$

Since $\Gamma$ is an invariant homogeneous graph, we have

$$
\sum_{b \in K}(f(a b x)-f(b a x))=0
$$


and therefore

$$
L \rho(x)=X=\frac{2 \lambda}{k} \sum_{a \in K}[f(x)-f(a x)]^{2}
$$

Now we consider

$$
\begin{aligned}
L f^{2}(x) & =\frac{1}{k} \sum_{a \in K}\left[f^{2}(x)-f^{2}(a x)\right] \\
& =\frac{2}{k} \sum_{a \in K} f(x)[f(x)-f(a x)]-\frac{1}{k} \sum_{a \in K}[f(x)-f(a x)]^{2} \\
& =2 \lambda f^{2}(x)-\frac{1}{k} \sum_{a \in K}[f(x)-f(a x)]^{2}
\end{aligned}
$$

Combining the above arguments, we have, for any positive $\alpha$, the following:

$L\left(\frac{1}{k} \sum_{a \in K}[f(x)-f(a x)]^{2}+\alpha \lambda f^{2}(x)\right) \leq 2 \alpha \lambda^{2} f^{2}(x)-\frac{(\alpha-2) \lambda}{k} \sum_{a \in K}[f(x)-f(a x)]^{2}$

Now we consider a vertex $v$ which achieves the maximum value, over all $x \in S$, for

$$
\frac{1}{k} \sum_{a \in K}[f(x)-f(a x)]^{2}+\alpha \lambda f^{2}(x)
$$

We have

$$
\begin{aligned}
0 & \leq L\left(\frac{1}{k} \sum_{a \in K}[f(v)-f(a v)]^{2}+\alpha \lambda f^{2}(v)\right) \\
& \leq 2 \alpha \lambda^{2} f^{2}(v)-\frac{\lambda(\alpha-2)}{k} \sum_{a \in K}[f(v)-f(a v)]^{2}
\end{aligned}
$$

This implies

$$
\frac{1}{k} \sum_{a \in K}[f(v)-f(a v)]^{2} \leq \frac{2 \lambda \alpha}{\alpha-2} f^{2}(v)
$$

for $\alpha>2$. Therefore for every $x \in V(\Gamma)$, we have

$$
\begin{aligned}
\frac{1}{k} \sum_{a \in K}[f(x)-f(a x)]^{2}+\alpha \lambda f^{2}(x) & \leq \frac{1}{k} \sum_{a \in K}[f(v)-f(a v)]^{2}+\alpha \lambda f^{2}(v) \\
& \leq \frac{2 \lambda \alpha}{\alpha-2} f^{2}(v)+\alpha \lambda f^{2}(v) \\
& \leq\left(\frac{2 \alpha}{\alpha-2}+\alpha\right) \lambda f^{2}(v) \\
& =\frac{\alpha^{2}}{\alpha-2} \lambda \max _{y} f^{2}(y)
\end{aligned}
$$

for any $\alpha>2$. The proof of Theorem 0 is complete. 
By taking $\alpha=4$ in Theorem 0 we have

Corollary 1. In an invariant homogeneous graph $\Gamma$ with edge generating set $K$ consisting of $k$ generators, suppose a function $f: V(\Gamma) \rightarrow \mathbf{R}$ satisfies

$$
L f(x)=\frac{1}{k} \sum_{a \in K}[f(x)-f(a x)]=\lambda f(x) .
$$

Then for all $x \in V(\Gamma)$

$$
\frac{1}{k} \sum_{a \in K}[f(x)-f(a x)]^{2} \leq 8 \lambda \sup _{y} f^{2}(y)
$$

\section{A HARNACK INEQUALITY FOR STRONGLY CONVEX SUBGRAPHS}

For strongly convex subgraphs, a similar but slightly different Harnack inequality for Neumann eigenvalues can be derived. The proof is based on the Neumann boundary condition and the assumption that the homogeneous graph is invariant.

Theorem 0. Suppose $S$ is a finite strongly convex subgraph in an abelian homogeneous graph $\Gamma$ with edge generating set $K$ consisting of $k$ generators. Let $f: S \rightarrow \mathbf{R}$ denote an eigenfunction associated with eigenvalue $\lambda$. Then the following inequality holds for $x \in S, a \in K$ and $a x \in S$ :

$$
[f(x)-f(a x)]^{2}+k \alpha \lambda f^{2}(x) \leq \frac{k \lambda \alpha^{2}}{\alpha-2} \sup _{y \in S} f^{2}(y)
$$

for any $\alpha>2$.

Proof: We consider, for some $g \in K$,

$$
\phi_{g}(x)=[f(x)-f(g x)]^{2}+k \alpha \lambda f^{2}(x)
$$

Let $\{z, a z\}$ be an edge in $S$ achieving the maximum value of $\phi_{g}(x)$ ranging over all $g \in K, x, g x \in S$.

Claim: $\phi_{g}(x) \leq \phi_{a}(z)$ for all $x, g x \in \delta S \cup S$.

Proof: We consider the following three possibilities:

Case 1: Suppose $g x \in S$ and $x \in \delta S$. We have, by using Lemma 1

$$
\begin{aligned}
\phi_{g}(x) & =[f(x)-f(g x)]^{2}+k \alpha \lambda f^{2}(x) \\
& =\left[\frac{1}{w} \sum_{b \in W} f(b x)-f(g x)\right]^{2}+k \alpha \lambda\left[\frac{1}{w} \sum_{b \in W} f(b x)\right]^{2}
\end{aligned}
$$


where $W=\delta x \cap S$ and $w=|W|$. Therefore

$$
\phi_{g}(x) \leq \frac{1}{w} \sum_{b \in W}[f(b x)-f(g x)]^{2}+\frac{k \alpha \lambda}{w} \sum_{b \in W} f(b x)^{2}
$$

From the definition of strongly convex subgraphs, $b x$ is adjacent to $g x$ for $b x \in W:$ Therefore, we have

$$
\phi_{g}(x) \leq \frac{1}{w} \sum_{b \in W} \phi_{g}(b x) \leq \phi_{a}(z)
$$

Case 2: Suppose $g x \in \delta S$ and $x \in S$.

$$
\begin{aligned}
\phi_{g}(x) & =[f(x)-f(g x)]^{2}+k \alpha \lambda f^{2}(x) \\
& =\left[f(x)-\frac{1}{w^{\prime}} \sum_{b \in W^{\prime}} f(b g x)\right]^{2}+k \alpha \lambda f^{2}(x) \\
& \leq \frac{1}{w^{\prime}} \sum_{b \in W^{\prime}}[f(x)-f(b g x)]^{2}+k \alpha \lambda f^{2}(x) \\
& \leq \frac{1}{w^{\prime}} \sum_{b \in W^{\prime}} \max _{g} \phi_{g}(x) \\
& \leq \phi_{a}(z)
\end{aligned}
$$

where $W^{\prime}=\delta g x \cap S$ and $w^{\prime}=\left|W^{\prime}\right|$. Here we use the fact that $b g x$ is adjacent to $x$ because of strong convexity.

Case 3: Suppose that $x, g x \in \delta S$. We define

$$
\begin{aligned}
& W_{1}=\{b: b x \in S, b g x \in S\} \\
& W_{2}=\{b: b x \in S, b g x \notin S\} \\
& W_{3}=\{b: b x \notin S, b g x \in S\}
\end{aligned}
$$


We set $w_{i}=\left|W_{i}\right|$ and $w=w_{1}+w_{2}+w_{3}$. Then

$$
\begin{aligned}
& \phi_{g}(x) \\
= & {[f(x)-f(g x)]^{2}+k \alpha \lambda f^{2}(x) } \\
= & {\left[\frac{w_{3}}{w} f(x)+\frac{1}{w} \sum_{b \in W_{1} \cup W_{2}} f(b x)-\frac{w_{2}}{w} f(g x)-\frac{1}{w} \sum_{b \in W_{1} \cup W_{3}} f(b g x)\right]^{2}+k \alpha \lambda f^{2}(x) } \\
\leq & \frac{1}{w}\left\{\sum_{b \in W_{1}}[f(b x)-f(b g x)]^{2}+\sum_{b \in W_{2}}[f(b x)-f(g x)]^{2}+\sum_{b \in W_{3}}[f(x)-f(b g x)]^{2}\right\} \\
& +\frac{k \alpha \lambda}{w}\left\{\sum_{b \in W_{1}} f^{2}(b x)+\sum_{b \in W_{2}} f^{2}(b x)+\sum_{b \in W_{3}} f^{2}(x)\right\}
\end{aligned}
$$

Suppose we can show that $b x \sim g x$ for $b \in W_{2}$ and $x \sim b g x$ for $b \in W_{3}$. Then by using Cases 1 and 2, we have $\phi_{g}(x) \leq \phi_{a}(z)$, as desired.

To see that $b x \sim g x$ for $b \in W_{2}$, we consider $b q z \notin S$ and $g x \in S, b x \in S$. By the definition of strong convexity, we have $b x \sim g x$. Also, $x \sim b g x$ for $b \in W_{3}$, since $b x \notin S$ and $x, b g x \in S$. The claim is proved.

Let $\phi(x)$ denote $\phi_{a}(x)$. We consider

$$
\begin{aligned}
L \phi(x) & =\frac{1}{k} \sum_{b \in K}[\phi(x)-\phi(b x)] \\
& \leq \frac{1}{k} \sum_{b \in K}\left[(f(x)-f(a x))^{2}-(f(b x)-f(a b x))^{2}\right]-\alpha \lambda \sum_{b \in K}\left[f^{2}(x)-f^{2}(b x)\right] \\
& =Y+Z
\end{aligned}
$$

where

$$
\begin{aligned}
Y= & \frac{1}{k} \sum_{b \in K}\left[(f(x)-f(a x))^{2}-(f(b x)-f(a b x))^{2}\right] \\
= & \frac{2}{k} \sum_{b \in K}(f(x)-f(a x)-f(b x)+f(a b x))(f(x)-f(a x) \\
& -\frac{1}{k} \sum_{b \in K}(f(x)-f(a x)-f(b x)+f(a b x))^{2} \\
\leq & \frac{2}{k}\left\{\sum_{b \in K}[f(x)-f(b x)]-\sum_{b \in K}[f(a x)-f(a b x)]\right\}(f(x)-f(a x)) \\
\leq & 2 \lambda[f(x)-f(a x)]^{2}
\end{aligned}
$$


and,

$$
\begin{aligned}
Z & =\alpha \lambda \sum_{b \in K}\left[f^{2}(x)-f^{2}(b x)\right] \\
& \leq 2 \alpha \lambda\left\{\sum_{b \in K}[f(x)-f(b x)] f(x)-\sum_{b \in K}[f(x)-f(b x)]^{2}\right\} \\
& \leq 2 \alpha \lambda\left\{k \lambda f^{2}(x)-\sum_{b \in K}[f(x)-f(b x)]^{2}\right\}
\end{aligned}
$$

Therefore, we have

$$
0 \leq L \phi(z) \leq 2 k \alpha \lambda^{2} f^{2}(z)-\lambda(\alpha-2) \sum_{a \in K}[f(v)-f(a v)]^{2}
$$

and

$$
[f(z)-f(a z)]^{2} \leq \frac{2 k \lambda \alpha}{\alpha-2} f^{2}(z)
$$

for $\alpha>2$. Therefore for all $x \in S, g \in K, g x \in S$, we have

$$
\begin{aligned}
{[f(x)-f(g x)]^{2}+k \alpha \lambda f^{2}(x) } & \leq[f(z)-f(a z)]^{2}+k \alpha \lambda f^{2}(z) \\
& \leq \frac{2 k \lambda \alpha}{\alpha-2} f^{2}(z)+k \alpha \lambda f^{2}(z) \\
& \leq\left(\frac{2 \alpha}{\alpha-2}+\alpha\right) \lambda k f^{2}(z) \\
& =\frac{\alpha^{2} \lambda k}{\alpha-2} \max _{y \in S} f^{2}(y)
\end{aligned}
$$

for any $\alpha>2$. The proof of Theorem 0 is complete.

By taking $\alpha=4$ in Theorem 0 we have

Corollary 2. Suppose $S$ is a strongly convex subgraph in an invariant abelian homogeneous graph $\Gamma$ with edge generating set $K$ consisting of $k$ generators. Let $f: S \rightarrow \mathbf{R}$ denote an eigenfunction associated with eigenvalue $\lambda$. Then for all $x \in S, a \in K$, we have

$$
[f(x)-f(a x)]^{2} \leq 8 k \lambda \sup _{y \in S} f^{2}(y)
$$

\section{EigenVAlues AND DiAmeters}

The Harnack inequality in previous sections can be used to derive the following eigenvalue inequality: 
Theorem 0. The eigenvalue $\lambda_{S}$ of a convex subgraph $S$ of an invariant homogeneous graph $\Gamma$ satisfies

$$
\lambda_{S} \geq \frac{1}{8 k D^{2}}
$$

where $k$ is the degree of $\Gamma$ and $D$ is the diameter of $S$.

Proof: Let $f$ denote an eigenfunction defined on $\delta S$ achieving $\lambda_{S}=\lambda$. We can choose $f$ such that

$$
\sup _{x \in S}|f(x)|=1=\sup _{x \in S} f(x)
$$

Let $u$ denote a vertex with $f(u)=\max _{x \in S} f(x)=1$ and let $v$ denote a vertex with $f(v)<0$. Such $v$ exists since $\sum_{x \in S} f(x)=0$. We now consider a shortest path $P$ in $S$ joining $u$ and $v$. We denote $P=\left(u=v_{0}, v_{1}, \cdots, v_{t}=v\right)$. Since the diameter of $S$ is $D$, we have $t \leq D$.

We consider

$$
S=\sum_{i=0}^{t-1}\left[f\left(v_{i}\right)-f\left(v_{i+1}\right)\right]^{2}
$$

By Theorem 2, we have

$$
S \leq 8 k \lambda D
$$

On the other hand, we have

$$
\begin{aligned}
S & =\sum_{i=0}^{t-1}\left[f\left(v_{i}\right)-f\left(v_{i+1}\right)\right]^{2} \\
& \geq \frac{1}{D}(f(u)-f(v))^{2} \\
& \geq \frac{1}{D}
\end{aligned}
$$

Therefore we obtain

$$
\lambda \geq \frac{1}{8 k D^{2}}
$$

and the proof of Theorem 3 is complete.

We remark that eigenvalue lower bounds of the form $\frac{c}{k D^{2}}$ are particularly useful for deriving polynomial approximation algorithms for enumeration problems since many families of combinatorial structures can often be represented as certain subgraphs of some appropriate homogeneous graphs. Eigenvalue lower bounds then translate to upper bounds (of the form $1 / \lambda_{S} \log n$ ) 
for the rate of convergence on the random walk problems which, under some mild conditions, can then be used to obtain randomized approximation algorithms. We note that the condition for strongly convex subgraph still pose quite severe constraints for some applications. In a subsequent paper [4], the convexity condition will be further relaxed and eigenvalue inequalities similar to that in Theorem 2 are derived for large families of graphs. The main methods are by using properties of the heat kernels and the approach is entirely different from that in this paper. Numerous applications in rapidly mixing Markov chains are further explored in [5].

\section{REFERENCES}

1. D. Aldous, On the Markov-chain simulation method for uniform combinatorial simulation and simulated annealing, Prob. Eng. info. Sci. 1 (1987) 33-46

2. L. Babai and M. Szegedy, Local expansion of symmetrical graphs, Combinatorics, Probability and Computing 1 (1991), 1-12.

3. F. R. K. Chung, Spectral Graph Theory, CMBS Lecture Notes, 1995, AMS Publication.

4. IF. R. K. Chung and S. -T. Yau, Eigenvalue inequalities for graphs and convex subgraphs, ppreprint.

5. F. R.IK. Chung, R. L. Graham and S. -T. Yau, On sampling in combinatorial structures, preprint.

6. Persi Diaconis and D. Stroock, Geometric bounds for eigenvalues of Markov chains, Ann. Appl. Prob. 1 36-61

7. S. T. Yau and Richard M. Schoen, Differential Geometry, (1988), Science Publication Co. (in Chinese)

University of Pennsylvania, Philadelphia, U. S. A. and Institute for Advanced Study, PRINCETON, U. S. A.

E-mail address: chung@math.upenn.edu

HARVARD UNIVERSITY, CAMBRIDGE, U. S. A.

E-mail address: yau@math.harvard.edu

Received November 8, 1994. 